viewed by a standing advisory committee, ${ }^{1}$ but the published list of interactions did not include ketoconazole or the related antifungal agent miconazole. The pharmacology of ketoconazole has been studied in patients and volunteers. ${ }^{2}$ Two volunteers were given warfarin and subsequently warfarin and ketoconazole; no potentiation of the anticoagulant effect of warfarin was found, and concentrations of ketoconazole were not altered.

No details of interaction between the two drugs in patients have been published to date, although one report has been made to the Committee on Safety of Medicines of an 84 year old man whose British comparative ratio rose to $4 \cdot 8$ when the two drugs were administered concomitantly; after ketoconazole was stopped the ratio fell to 1.4. The manufacturers, Janssen Pharmaceutical Limited, have not had any report of interaction between these drugs. In the light of these cases, when ketoconazole is prescribed for patients being treated with warfarin the precautions currently advised for patients being treated with broad spectrum antibiotics ${ }^{1}$ should be taken-namely, more frequent monitoring of anticoagulant control and reduction of the warfarin dosage by one third.

1 Standard Advisory Committee for Haematology of the Royal College of Pathologists. Drug interaction with coumarin derivative anticoagulants. Br Med f $1982 ; 285: 274-5$.

${ }^{2}$ Brass C, Galgiani J, Blaschke T, Defelice R, O'Reilly R, Stevens D. Disposition of ketoconazole, an oral antifungal, in humans. Antimicrob Agents Chemother 1982;21:151-8.

(Accepted 14 September 1983)

Haematology Department, Royal South Hants Hospital, Southampton SO9 4PE

ALASTAIR G SMITH, MRCP, MRCPATH, consultant

\section{Acute brain stem stroke during neck manipulation}

Manipulation of the neck as a cause of infarction of the brain or spinal cord is thought to be rare, ${ }^{1}$ but this may not be so. ${ }^{2}$ We report the occurrence of brain stem stroke during neck manipulation.

\section{Case report}

A 31 year old man in good health consulted an osteopath for relief of neck pain. Neck manipulation by the osteopath proceeded no further than rotation of the head to the right. Immediately the patient complained of tingling of the right side of the face, right arm, and right leg followed by slurred speech, double vision, and difficulty in closing the left eye. He was examined at this hospital one hour after the onset of his stroke. There was an incomplete left facial palsy of the lower motor neurone type, diplopia and coarse nystagmus on looking to the right, depression of sensation to pinprick and light touch on the right side of the face, dysarthria, and ataxia of the right arm and leg. The tendon reflexes were not exaggerated, and both plantar responses were downgoing. There was no limb weakness but he was unable to stand because of unsteadiness. A bruit was heard over the left carotid artery. Other examination findings were normal.

Full blood count, urea and electrolyte concentrations, liver function values, blood sugar and lipid concentrations, electrocardiogram, results of cardiac ultrasound, and radiographs of skull, chest, and cervical spine were normal. A CT brain scan showed a poorly defined area of low attenuation near the fourth ventricle in the cerebellum but was otherwise normal. An arch aortogram one week after the stroke showed an irregular and narrowed left vertebral artery, with occlusion at the site of its passage between the first and second cervical vertebrae. Some collateral circulation was established (figure). These changes were consistent with a traumatic thrombosis of this vessel. Angiographic appearances of the other cranial vessels were normal.

The patient improved without treatment. Ten days after the stroke he had slight nystagmus to the right, slight ataxia of the right arm and leg, but a pronounced left facial palsy. Six months after the stroke the facial palsy was barely noticeable and he had returned to his job as a fork lift driver.

\section{Comment}

This patient apparently had a traumatic occlusion of his left vertebral artery during rotation of the neck, resulting in acute brain stem and cerebellar ischaemia and probable infarction. Careful review of his previous health, family history, and other possible trauma to the neck was unrewarding.

Thrombosis of this vessel during neck manipulation has been recognised as a cause of brain stem, cerebellar, or spinal cord infarction since $1947,{ }^{3}$ but by 1981 only 52 patients had been reported. ${ }^{1}$ A recent American survey, however, yielded 360 additional cases. ${ }^{2}$ In Germany the Society of Manipulative Medicine has kept a registry of such cases. ${ }^{2}$ This injury tends to occur in the relatively young (mean aged 37 years), the mortality tends to be high, and anticoagulants in the period immediately after the stroke have therefore been recommended. ${ }^{1}$

This injury usually occurs at the atlantoaxial level and is determined by the anatomical relations of the vertebral arteries. These

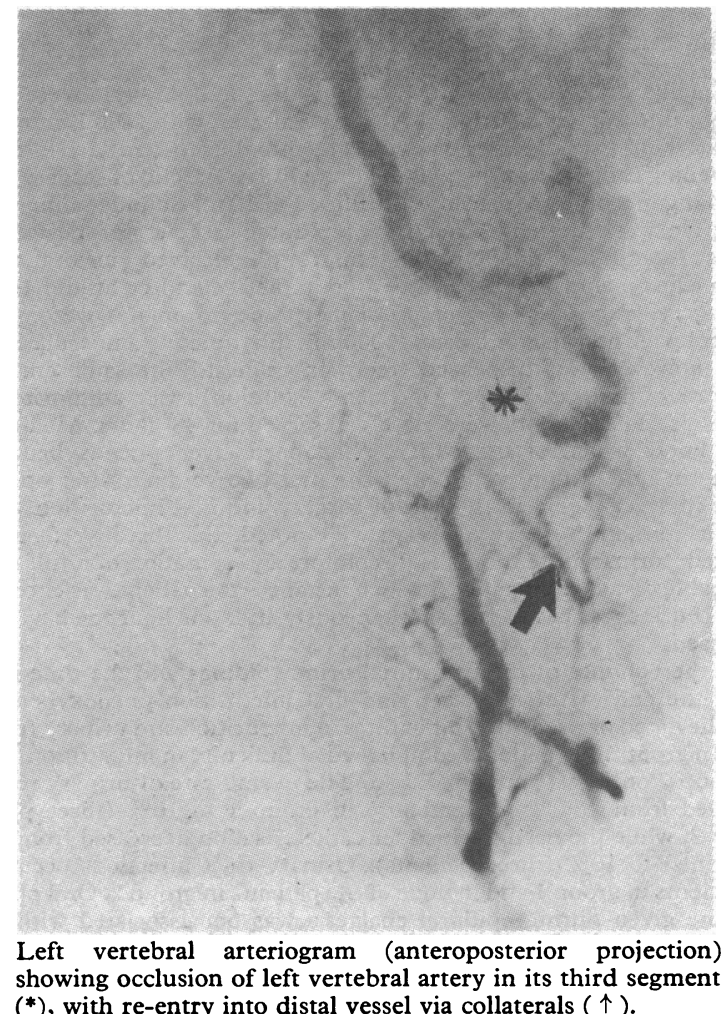

vessels are relatively fixed in the transverse foramina between the first and second cervical vertebrae. The atlantoaxial joint is the site of rotation and of some tilting of the head and neck. These factors favour the stretching, temporary occlusion, or thrombosis of the vertebral arteries during manipulation of the neck. ${ }^{4}$ Other manoeuvres associated with head turning have also caused strokes-for example, falls, painting the ceiling, yoga, gymnastics, archery, and head turning while driving a car. ${ }^{1}$ To our knowledge there have been no other reports from Britain of stroke during neck manipulation. In view of the American and German experience ${ }^{2}$ we suspect that such injury may have gone unnoticed or unreported.

${ }^{1}$ Sherman DG, Hart RG, Easton JD. Abrupt change in head position and cerebral infarction. Stroke $1981 ; 12: 2-6$.

${ }^{2}$ Robertson JT. Neck manipulation as cause of stroke. Stroke 1982;13: 260-1.

${ }^{3}$ Pratt-Thomas HR, Berger KE. Cerebellar and spinal injuries after chiropractic manipulation. $\mathcal{F} A M A 1947 ; 133: 600-3$.

(Accepted 23 September 1983)

Frenchay Hospital, Bristol BS16 1LE

TAWFIQUE K DANESHMEND, MRCP, registrar in neurology

RICHARD LANGTON HEWER, FRCP, consultant neurologist

JOHN R BRADSHAW, FRCR, consultant radiologist

Correspondence to: $\mathrm{Dr}$ T K Daneshmend, Department of Medicine, Southmead Hospital, Bristol BS10 5NB. 